Int. J. Dev. Biol. 56: 747-753 (2012)

doi: $10.1387 / \mathrm{ijdb} .120017 \mathrm{sh}$

\title{
Expression analysis of the polypyrimidine tract binding protein (PTBP1) and its paralogs PTBP2 and PTBP3 during Xenopus tropicalis embryogenesis
}

\author{
MAUD NOIRET ${ }^{1,2}$, YANN AUDIC $^{1,2}$ and SERGE HARDY*,1,2 \\ ${ }^{1}$ CNRS, UMR 6290, Institut Génétique et Développement de Rennes and \\ ${ }^{2}$ Université Rennes 1, UEB, Biosit UMS 3480, Faculté de Médecine, Rennes, France
}

\begin{abstract}
The PTB (polypyrimidine tract binding protein) family of RNA-binding proteins plays a critical role in development through the regulation of post-transcriptional events. We have determined expression patterns of the three members of this gene family ptbp1, ptbp2 and ptbp3 during Xenopus tropicalis embryogenesis using whole-mount in situ hybridization. Our results show that each paralog presents a unique pattern of expression. ptbp 1 is the prevalent maternal mRNA and is differentially expressed in the three germ layers. Later in development, it is widely expressed in the embryo including the epidermis, the dermatome, the intermediate mesoderm, the lateral plate mesoderm and the neural crest. ptbp2 expression is restricted to the nervous system including the brain, the neural retina and the spinal cord and the intermediate mesoderm. In addition to being expressed in erythroid precursors, ptbp 3 is present in specific subdomains of the brain and the spinal cord, as well as in the posterior part of the notochord, suggesting it may play a role in the patterning of the nervous system. In the eye, each of the three genes is expressed in a specific structure which emphasizes their non-redundant function during development. Strickingly, our experiments also revealed that none of the three paralogs was expressed in the myotome, suggesting that the absence of PTB activity is a key determinant to display myotomal splicing patterns.
\end{abstract}

KEY WORDS: RNA binding protein, Xenopus, PTB

The polypyrimidine tract binding protein (PTB) also known as PTBP1 or hnRNPI is one of the most investigated RNA-binding protein (RBP) in vertebrates. It is a key factor in the control of RNA metabolism (reviewed in Sawicka et al., 2008) as it was shown to regulate mRNA alternative splicing events (Sauliere et al., 2006; Wagner and Garcia-Blanco, 2001), 3' end-processing (Hamon et al., 2004), mRNA stability (Knoch et al., 2004), mRNA localization (Cote et al., 1999) and internal ribosome entry-site dependant translation (Ohno et al., 2011). Its activity upon splicing regulation is now well documented and while it was first essentially described as a repressor factor, high throughput studies showed that it can promotes both exon repression and activation depending of its binding location relative to the regulated exon (Llorian et al., 2010). In vertebrates, PTBP1 is an essential protein. Its knockdown in Xenopus embryos disturbs the dorsal fin morphogenesis with the apparition of blister-like structures and causes the subsequent death of tadpoles (Le Sommer et al., 2005). In mouse, PTBP1 is required for embryonic development before gastrulation and its knockout results in lethality shortly after implantation (Shibayama et al., 2009; Suckale et al., 2011). In Drosophila, mutations in the hephaestus gene that encode the PTBP1 orthologue (dmPTB) have pleiotropic effects including oogenesis, spermatogenesis wing development and cardiogenesis defects. Neurogenesis is also suppressed in null embryos (Wesley et al., 2011).

PTBP1 is generally described as a widely expressed factor in adult tissues and accordingly it is present in most of the cell lines studied (Wang et al., 2008). However, its amount can strongly vary from tissue to tissue or depending on the cellular differentiation states. For example, while it is highly expressed in proliferating neuronal and muscle cells, its level is strongly reduced with the onset of neuronal or muscle differentiation (Makeyev et al., 2007,

Abbreviations used in this paper: CMZ, ciliary marginal zone; FXR1, fragile X mental retardation syndrome-related protein 1; NMD, nonsense-mediated decay; PTB, polypryrimidine tract binding protein; RBP, RNA binding protein; UFE, unfertilized egg.

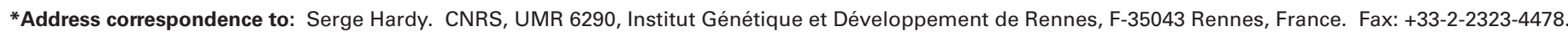
e-mail: serge.hardy@univ-rennes1.fr
} 
Boutz et al., 2007b, Boutz et al., 2007a). The level of PTBP1 may strongly influence its activity on RNA splicing. A genome wide analysis in HeLa cells indicates that PTBP1 knockdown activates the inclusion of muscle and brain specific exons in agreement with the low PTBP1 level in both of these tissues (Llorian et al., 2010). In Xenopus embryos, it was formally demonstrated that the relative level of PTBP1 is determinant for the tissue-specific switches of $\alpha$-tropomyosin mRNA isoforms. Its knockdown in the epidermis resulted in the apparition of a myotomal isoform with the splicing of a proximal 3'-end exon while its overexpression in the myotome generated the skipping of the proximal 3' end exon and a switch towards the non-muscle isoform (Le Sommer et al., 2005). The amount of PTBP1 is therefore crucial for its activity.

PTBP1 belong to a family of RNA binding proteins that include two additional members: PTBP2 also known as brPTB or nPTB and PTBP3 originally called ROD1. PTBP2 was first described in neuronal cells but it is also expressed in testis and at lower levels in skeletal muscles and heart (Lillevali et al., 2001; Xu and Hecht, 2007). It is involved in the regulation of alternative splicing and accordingly in differentiated neurons it controls numerous neural alternative splicing patterns (Boutz et al., 2007b). Studies with reporter gene models indicate that PTBP2 has a weaker repressive activity than PTBP1 (Ashiya and Grabowski, 1997). In testis, PTBP2 was shown to stabilize some specific mRNAs (Xu and Hecht, 2007; Xu and Hecht, 2008). PTBP3 is the least studied of the three paralogs. It is considered to be predominantly expressed in embryonic and adult hematopoietic organs and it was shown that its overexpression in myeloid cells blocks both megakaryocytic and erythroid differentiation (Yamamoto et al., 1999). However, to date no molecular function was assigned to this RBP.

In addition to share structural homology, the three paralogs have functional redundancy. Indeed, in HeLa cells the knockdown of PTBP1 has limited effect due to the strong up-regulation of PTBP2 which replaces many of the functions of PTBP1 in these cells. Accordingly, only the simultaneous inhibition of both PTBP1 and PTBP2 modify strongly the transcriptome of these cells. A functional link between the three paralogs is also illustrated by the fact that they cross-regulate each other. Indeed, in addition to regulate its own level by mediating exon skipping, leading to nonsense-mediated decay (NMD), PTBP1 may also down regulate PTBP2 via a similar mechanism. Reduced level of PTBP1 or PTBP2 is also required in HeLa cells to express a functional ptbp3 mRNA (Spellman et al., 2007).

Here, we describe for the first time the spatio-temporal expression of ptbp 1 and its paralogs ptbp2 and ptbp3 during Xenopus tropicalis embryogenesis. Our results show that each transcript presents a unique temporal and spatial expression pattern. Strikingly, we observed that ptbp1 which is considered as a generally expressed RBP is present in only two of the primitive germ layers, the ectoderm and the mesoderm. Later in development it is present in most mesodermal derivatives but not the myotome. ptbp2 and ptbp3 are also not expressed in the myotome suggesting that the absence of any PTB activity is a major determinant to display myotomal-specific splicing pattern. Our data confirm that ptbp2 expression is restricted to the developing brain and the spinal cord underscoring its unique function in the development of the nervous system. Our results also show that in addition to be expressed in erythroid precursors, ptbp3 is present in several non-hematopoietic tissues including specific subdomains of the brain and the spinal cord suggesting it may have extended function during embryogenesis and more specifically may play a role in the patterning of the nervous system.

\section{Results}

\section{Phylogenetic analysis of the Xenopus PTB family}

The full length cDNA sequences for Xenopus tropicalis ptbp1 (NM_001011140), ptbp2 (NM_001079011) and ptbp3 (NM_001127046) were obtained from Genbank database. The translated sequences were used to create a phylogenetic tree showing the evolutionary relationship between these proteins and their orthologs in Drosophila and different vertebrates (Fig. 1). In addition to confirm that the proteins studied are the true orthologs of the three paralogs described in other species, our analysis showed that the PTB proteins fell into two families, one corresponding to PTBP1 and PTBP2, the other corresponding to PTBP3.

\section{Temporal expression of PTBP1, PTBP2 and PTBP3 during early Xenopus development}

The temporal expression pattern of ptbp1, ptbp2 and ptbp3 mRNAs were determined by RT-qPCR and are presented in Fig. 2. The mRNAs expression profiles were normalized to the

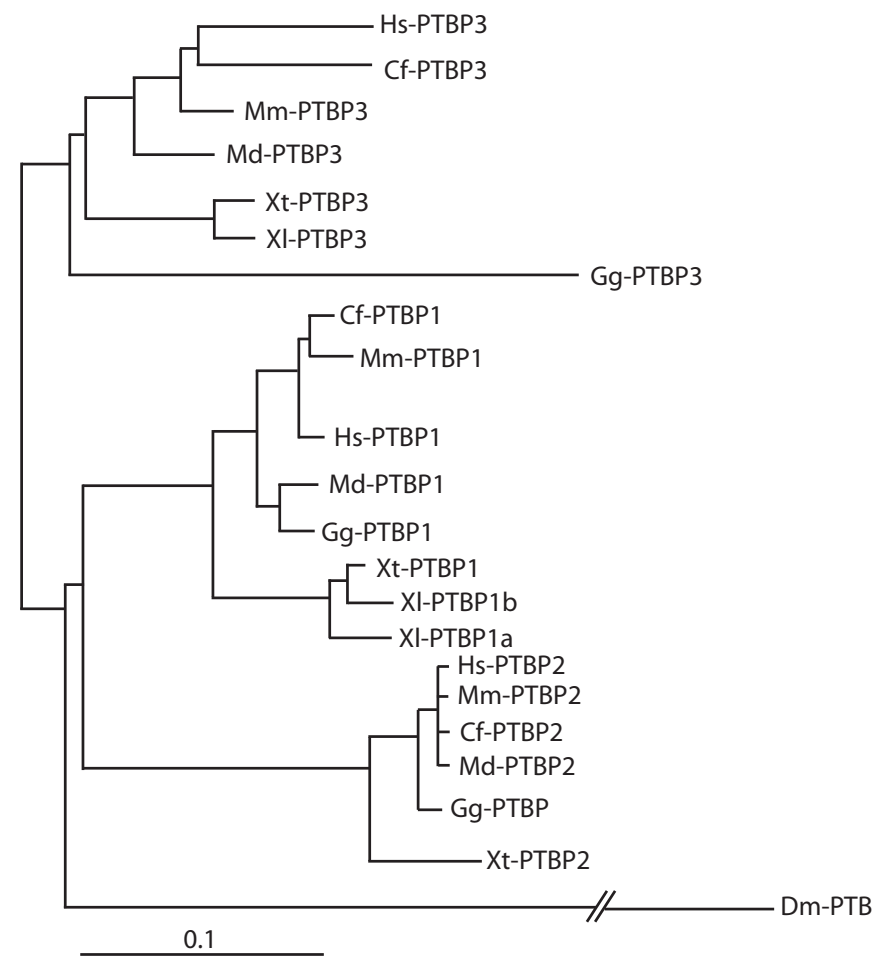

Fig. 1. Phylogenetic analysis of polypyrimidine tract binding protein (PTB) family members. The phylogenetic tree was constructed with the Neighbor-Joining method as described in the material and methods section. Parallel slanted lines designate interruptions of the normal scale and correspond to a distance of 0.5. The scale bar represents the number of amino acids substitutions. The abbreviations used are Cf: Canis familiaris, Dm: Drosophila Melanogaster, Gg, Gallus gallus; Hs, Homo sapiens; Md, Monodelphis domestica; $M m$, Mouse musculus; XI, Xenopus laevis; Xt, Xenopus tropicalis. 
endogenous odc mRNA of each embryonic stage and to unfertilized eggs (UFE) (Fig. 2A). Similar results were obtained when the normalization was realized with an exogenous luciferase reporter mRNA (data not shown). The three mRNAs are detectable in unfertilized eggs indicating their maternal origin, however after fertilization each mRNA presents a specific expression pattern. For ptbp1 (top panel), a slight decrease was first observed, the minimum being reached around stage 7 . Then, consequently to the zygotic transcription activation, ptbp 1 transcripts raised to reach from stage 20 a three-fold-higher level than in UFE. For ptbp2 and ptbp3 mRNAs (medium and bottom panels), a decrease was also observed but the minimum was reached at stage 11 suggesting that the zygotic transcription resumes later for these two genes. From stage 16, ptbp2 transcripts increased progressively to reach a 2 fold-higher level at stage 34 than in UFE. On the other hand, ptbp3 mRNAs raised strongly during early development to achieve a 10 fold higher level.

To compare the expression levels of the three paralogs, the levels of ptbp2 and ptbp3 mRNAs were also normalized to that of ptbp1 transcripts (Fig. 2B). In UFE, the amounts of ptbp2 and ptbp3 transcripts were respectively 3 and 11 times lower than ptbp1 mRNAs, indicating that ptbp1 is the prevalent maternal mRNA. During development, the level of ptbp2 transcripts is kept 3 to 6 times lower than ptbp 1 while because of the strong increase in expression of ptbp3, the difference between ptbp3 and ptbp 1 transcripts is strongly reduced.

\section{Spatial expression of PTBP1, PTBP2 and PTBP3 during early embryogenesis}

The spatial expression pattern of ptbp1, ptbp2 and ptbp3 mRNAs during early embryogenesis was studied by whole-mount in situ hybridization (Fig. 3). Since the three paralogs present a $70 \%$ nucleotide sequence identity in the coding region, sequences corresponding to the 3' UTR were chosen for preparation of probes. Sense probes were also used to verify specificity in the experiments (data not shown). As expected from the RT-qPCR analysis, ptbp1 was maternally expressed in UFE (Fig. 3A panel a). However, the labeling was present only in the animal pole. In situ hybridization of bisected UFE confirmed this spatial distribution (Fig. 3A panel a') suggesting that ptbp1 may specifically be expressed in this region. At the blastula stage, the expression was still detected mainly in the animal hemisphere but was expanded also through the marginal zone (Fig. 3A panel b and b'). During gastrulation, the ptbp 1 labeling covered the entire embryo except for the yolk plug, indicating a strong expression in ectoderm (Fig. $3 \mathrm{~A}$ panels $\mathrm{C}$ and $\left.\mathrm{c}^{\prime}\right)$. The absence of labeling in the yolk plug was not the consequence of poor probe penetration because a similar pattern was observed with bisected gastrula embryos (Fig. $3 \mathrm{~A}$ panel c") while the endodermal sox $17 \mathrm{~b}$ marker (D'Souza et al., 2003) strongly stained this region (Fig. $3 A$ panels $f$ and $f^{\prime}$ ). Analysis of bisected gastrula stages also revealed expression in the mesoderm and no labeling in the deep endodermal cells (Fig. $3 A$ panels $C$ " and C"') showing therefore a differential expression of PTBP1 between the three germ layers. At the neurula stages (stage 16-18) the ectodermic staining persisted associated with an accentuation of the expression in the neural plate region (Fig. 3 A panel d). Shorter detection times showed that the labeling was spatially present in the anterior and posterior neural plates border and the neural crests regions (Fig. 3A panels d' and d")
A
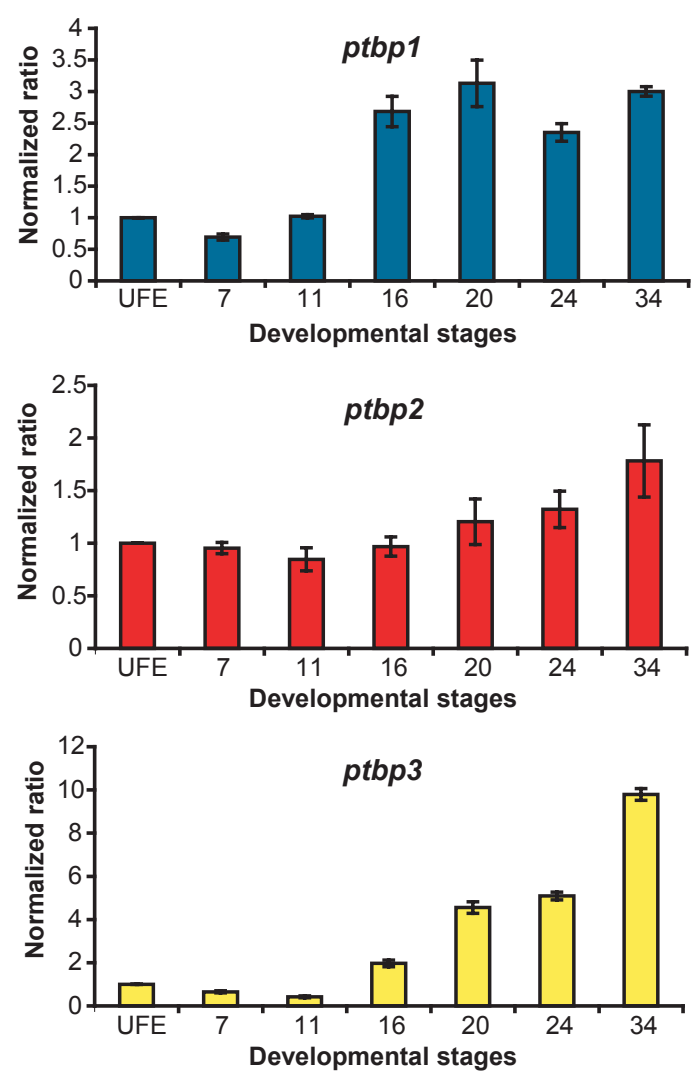

B

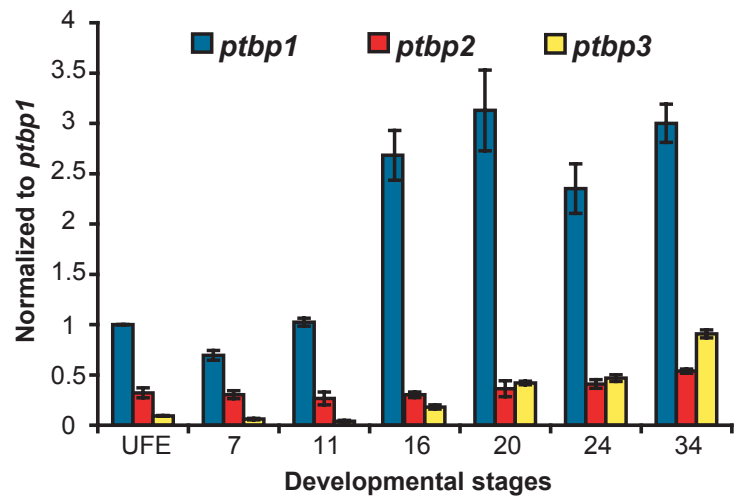

Fig. 2. Temporal expression profiles of polypyrimidine tract binding protein (PTB) genes during Xenopus development. (A) mRNA expression profiles normalized to the endogenous odc mRNA of each embryonic stage. Results are expressed with the normalized expression of the gene of interest in the Unfertilized Eggs (UFE) arbitrarily set to 1. (B) mRNA expression profiles normalized to ptbp1 mRNA. The amount of ptbp2 and ptbp3 $m R N A$ s presented in (A) were normalized to that ofptbp1 transcripts in UFE arbitrarily set to 1. (A,B) The horizontal axes represent the UFE and embryos at the different developmental stages determined according to Nieuwkoop and Faber (Nieuwkoop and Faber, 1956).

which is consistent with data previously reported in $X$. laevis (Tamanoue et al., 2010), At the early tailbud stage (stage 25, Fig. $3 \mathrm{~A}$ panel e), in addition to the strong epidermal signal, an enhanced expression was detected in the branchial arches, eyes, otic placodes and pronephros. In contrast to ptbp1, ptbp2 and 
ptbp3 showed no staining in UFE and blastula (Fig. 3 B,C panels a-c) suggesting that most of the maternal functions of this RBP family are performed by PTBP1.

Consistent with previous studies in mouse (Lillevali et al., 2001), the expression of ptbp2 coincided with the formation of the nervous system and ptbp2 mRNAs were first detected in the neural plate of late neurula stage (stage 18, Fig. 3B panel $d$ and d') with an enhanced expression in the anterior region that will form the brain. In early tailbud, with the progression of neurulation, ptbp2 transcripts mRNAs were specifically expressed throughout the whole fore, mid- and hindbrain as well as in the spinal cord (Fig. 3B panels e and e'). A strong labeling of the eyes was also observed (Fig. 3B panel e).

For ptbp3, a slight and general expression started at the neurula stage (stage 14) (data not shown) suggesting it is expressed in the ectoderm from this stage. At early tailbud stage (stage 22, Fig. 3C panels $d$ and d'), an epidermal general labeling was still present. A low level of ptbp3 expression was also consistently present in the ventral blood islands (vbi), the primary site of hematopoiesis. This expression domain is in agreement with a function of PTBP3 in erythroid differentiation (Yamamoto et al., 1999). At later tailbud stages, in addition to the epidermal staining, accentuated expression was observed in the eyes, the branchial arches and the notochord (Fig. 3C panel e) while the ventral labeling was no longer observed (Fig. 3C panel e').

\section{Spatial expression of PTBP1, PTBP2 and PTBP3 in late tailbud embryos}

To further explore the expression of ptb genes in the later stages of embryogenesis, transversal and longitudinal sections of late tailbud (stage 34) that were subjected to whole-mount in situ hybridization were realized and analyzed (Fig. 4). With the three probes a staining was observed in the ventral region of the head that may correspond to some non specific retention of the probes since similar labeling was obtained with the sense probe (data not shown). For ptbp1 (Fig. 4A), a specific labeling in the head was observed in the eyes, mandibular, hyodial and branchial arches and otic vesicles (Fig. 4A panels a,b,c,d,e and g). In the eyes, the expression was restricted to the retinal cilliary marginal zone (CMZ) (Fig. 4A panels b,c and e), the region where retinal stem cells proliferate and differentiate. In transversal troncal sections, a strong ptbp1 expression domain was observed in the somatic layer of lateral plate mesoderm as well as in the intermediate mesoderm that includes both pronephros and mullerian duct (Fig. 4A panels $h$ and i). This labelling was not apparent with whole-mount observations because of the general epidermal staining. Ventrally, ptbp 1 transcripts are specifically detectable in the ventral aorta while no labeling was observed in the heart (Fig. 4A panels $f$ and
A

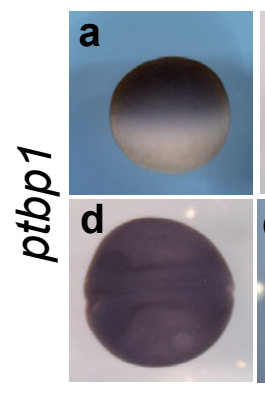

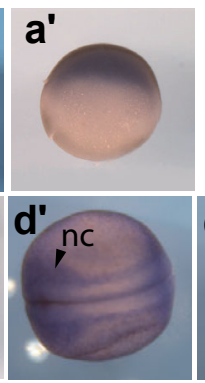
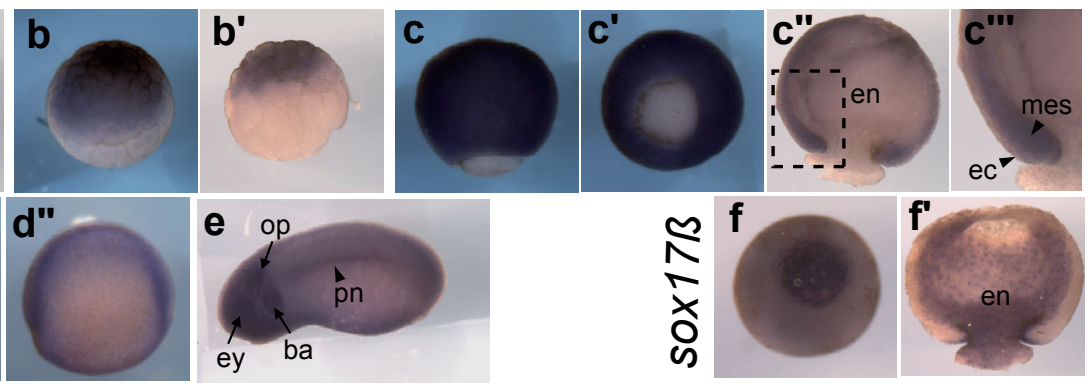

B
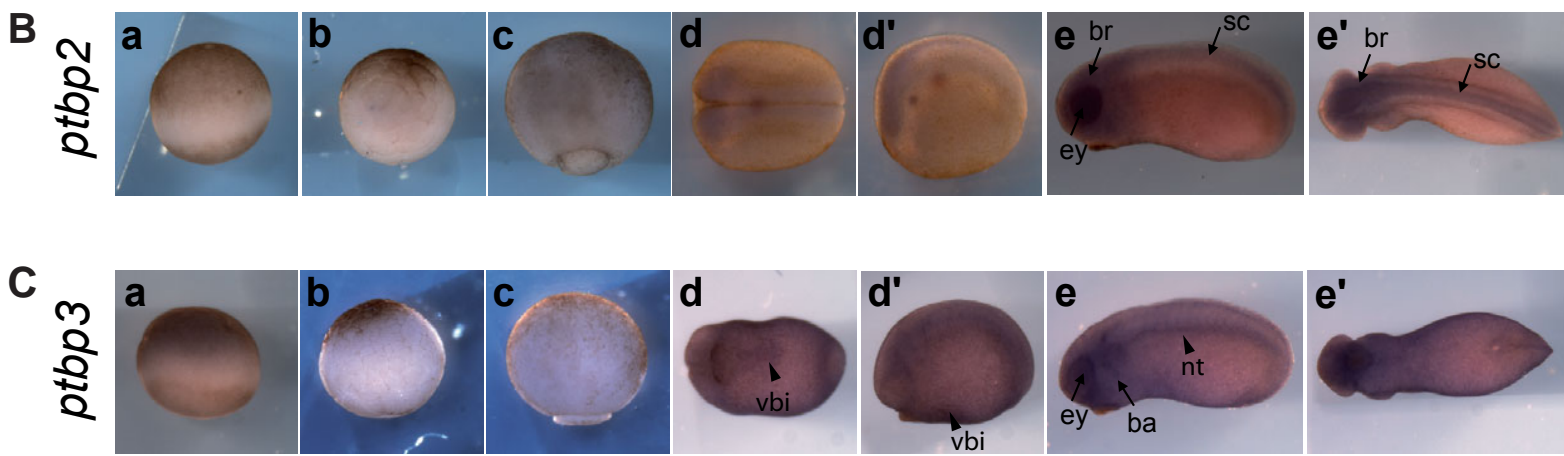

Fig. 3. Spatial expression profiles of polypyrimidine tract binding protein (PTB) genes during early Xenopus development. Whole-mount in situ hybridization was performed to study the spatial expression of ptbp1, ptbp2 and ptbp3 at different developmental stages. (A). ptbp1 (a) lateral view of an unfertilized egg (UFE), (a') UFE bisected through the animal-vegetal axis, (b) lateral view of a blastula, (b') blastula bisected through the animalvegetal axis, (c) lateral view of a gastrula, $\left(c^{\prime}\right)$ vegetal view of panel $c,\left(c^{\prime \prime}\right)$ gastrula bisected through the animal-vegetal axis, $\left(c^{\prime \prime \prime}\right)$ higher magnification view of the boxed area in panel $c^{\prime \prime},\left(d-d^{\prime}\right)$ dorsal views of neurula stages with two different detection times, $\left(d^{\prime \prime}\right)$ lateral view of panel $d^{\prime}$, (e) lateral view of an early tailbud embryo at stage 24. sox $17 \beta$ (f) vegetal view of a gastrula, (f') gastrula bisected through the animal-vegetal axis. (B) ptbp2 (a) lateral view of an UFE, (b) lateral view of a blastula, (c) lateral view of a gastrula, (d) dorsal view of neurula stage, (d') lateral view of panel d, (e) lateral view of an early tailbud embryo at stage 24, (e') dorsal view of panel e. (C) ptbp3 (a) lateral view of an UFE, (b) lateral view of a blastula, (c) lateral view of a gastrula, (d) ventral view of an early tailbud embryo at stage 20, (d') lateral view of panel d, (e) lateral view of an early tailbud embryo at stage 24 , (e') ventral view of panel e. Abbreviations used are: ba, branchial arches; br, brain; ec, ectoderm; en, endoderm; ey, eye; mes, mesoderm; nc, neural crests; nt, notochord; op, otic placode; pn, pronephros, vbi, ventral body island; sc, spinal cord. 


\section{A \\ $\frac{2}{2}$}
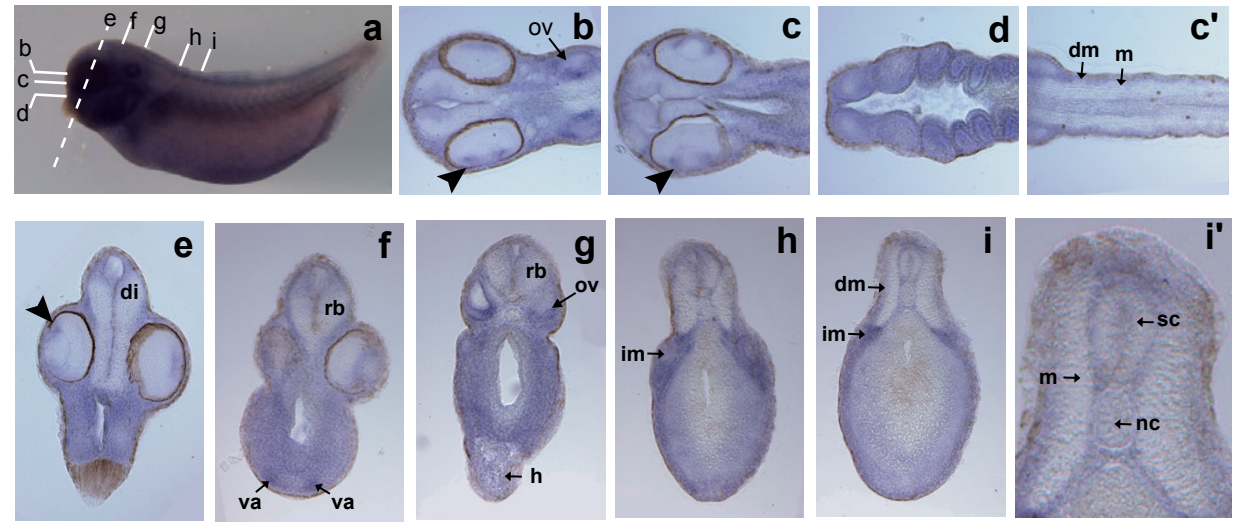

B
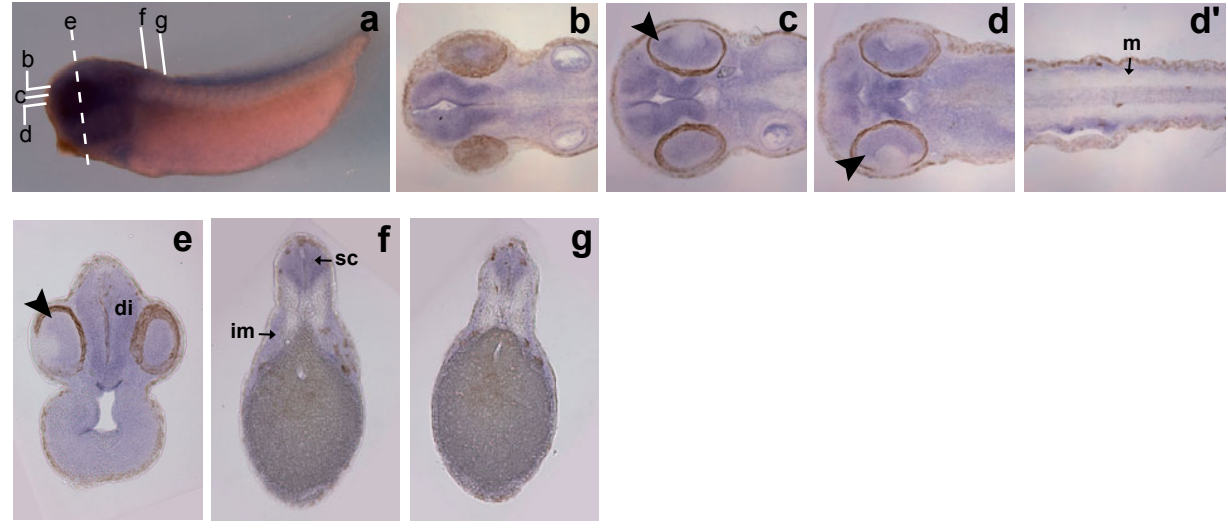

C
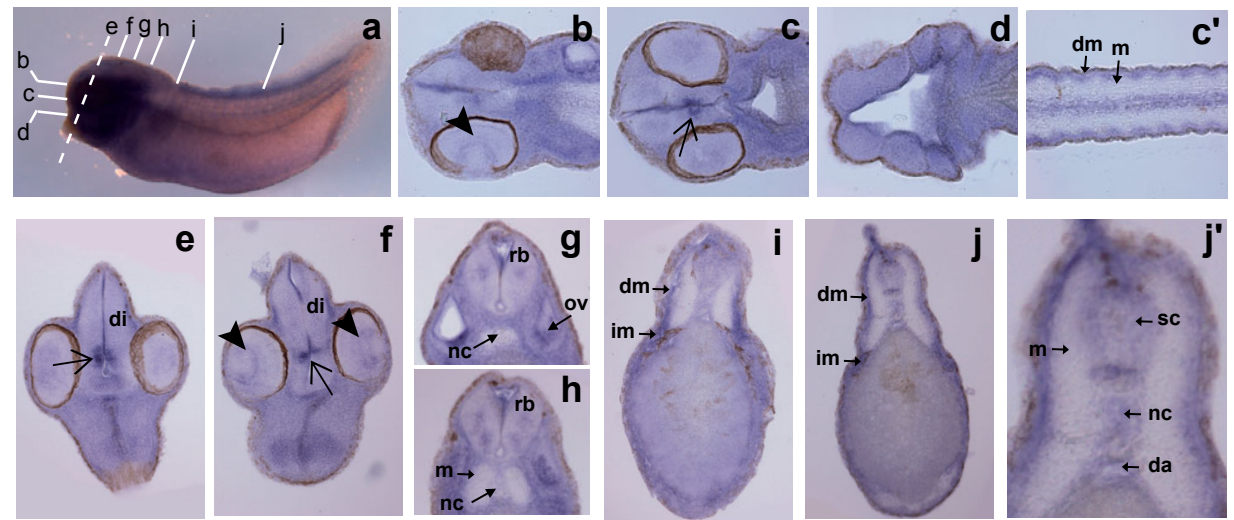

Fig. 4. Spatial expression profiles of polypyrimidine tract binding protein (PTB) genes in late tailbud embryos. Whole-mount in situ hybridization followed by longitudinal and transverse sections were performed to examine the spatial expression of ptbp1, ptbp2 and ptbp3 in late tailbud embryos. (A) ptbp1 (a) lateral view of a stage 34 embryo, the white lines indicate the level of transverse and longitudinal sections shown in panels $b-i,(b-d)$ longitudinal sections of the head along the dorso to ventralaxis, ( $\left.c^{\prime}\right)$ longitudinal section of the trunk realized at the same level as panel $c$, (e-i) transverse sections along the anterior to posterior axis, (i') higher magnification view of panel i. Black arrowheads point to the $C M Z$ of the retina (B) ptbp2 (a) lateral view of a stage 34 embryo, the white lines indicate the level of transverse and longitudinal sections shown in panels $b-g,(b-d)$ longitudinal sections of the head at the level of the brain, $\left(d^{\prime}\right)$ longitudinal section of the trunk realized in the same plan as panel $d,(e-g)$ transverse sections of head and trunk. Black arrowheads point to the neuronal retina. (C) ptbp3 (a) lateral view of a stage 34 embryo, the white lines indicate the level of transverse and longitudinal sections shown in panels b-j, (b-d) longitudinal sections of the head along the dorso to ventral axis, (c') longitudinal section of the trunk realized at the same level as panel $c,(e, f)$ transverse sections of the head at the level of the diencephal. $(g, h)$ higher magnification views of transverse sections of the head at the level of the rhombocephal, $(i, j)$ transverse sections of the trunk, (j') higher magnification view of panel j. Black arrowheads and arrows point respectively to the posterior lens and the hypothalamic labeling. Abbreviations used are: da, dorsal aorta; di, diencephal; $d m$, dermatome; $h$, heart; im, intermediate mesoderm; $m$, myotome, nc, notochord, ov, otic vesicle; rb, rhombocephal; sc, spinal cord; va, ventral aorta. g). Dorsally, ptbp1 mRNAs were expressed in the dermatome and the area surrounding the notochord and the spinal cord that contains the sclerotome and neural crest cells migrating ventrally, a strong staining was also present in neural crest cells migrating dorsally into fin (Fig. 4A panel i'). No ptbp1 labeling was present in the myotome all along the antero-posterior axis (Fig. 4A panel i,i' and $\left.c^{\prime}\right)$. An antisense control probe against the somitic marker fxr1 (Bourdelas et al., 2004) confirmed that the absence of labeling is not due to poor probe penetration in this tissue (data not shown).

In agreement with the pictures of whole-mount in situ hybridization, ptbp2 mRNAs were strongly expressed throughout the whole fore, mid- and hindbrain as well as in the spinal cord (Fig. $4 \mathrm{~B}$ panels $a, b, e, f$ and $g$ ). No specific regionalization of the staining was noticed within these tissues which is in accordance with a general function of PTBP2 in neuronal development. The mutually exclusive pattern of expression of ptbp1 and ptbp2 in the diencephal is clearly observed (Compare panels e of Fig. 4A and $4 \mathrm{~B}$ ) and is in agreement with the model that in brain, ptbp2 mRNA expression is relieved in absence of PTBP1 activity (Boutz et al., 2007b; Makeyev et al., 2007). In accordance with a specific expression in differentiated neuronal cells, in the eyes, the labeling was limited to the neural retina and is excluded from the CMZ (Fig. $4 \mathrm{~B}$ panels $\mathrm{C}$ and $\mathrm{d}$ ). A faint expression domain was also present in the intermediate mesoderm (Fig. 4B panel f).

Among ptb genes, ptbp3 presented unique localizations in the central nervous system. It was weakly expressed in the forebrain except for a strong labeling in a region corresponding to the dorsal hypothalamus (Fig. 4C panels c, e and f). Interestingly, ptbp3 positive cells were present in the ventricular area (Fig. 4C panel e) that corresponds to a zone of neuroepithelium proliferation. 
Discrete and symmetric areas of labeling were observed in the ventral rhombocephal. In the anterior part, the expression area corresponded to two symmetric nuclei that expand then posteriorly into four patches (Fig. 4C panels $g$ and $h$ ). In the trunk, the spinal cord was slightly labeled with a marked expression in the dorsal roof plate and the ventral floor plate. (Fig. $4 \mathrm{C}$ panels j and j'). ptbp3 is also expressed in the eyes in the region that correspond to the posterior lens and in the otic vesicles (Fig. 4C panels b, $\mathrm{f}$ and $\mathrm{g}$ ). In transversal troncal sections, ptbp3 transcripts were present in the notochord while they were not detected in the anterior notochord adjacent to the rhombocephal (Compare Fig. 4C panels i-j and $g-h)$. Ventrally to the notochord a specific labeling is present in the dorsal aorta (Fig. 4C panel j'). An accentuated expression of ptbp3 mRNAs is also detected in the dermatome while as already noticed for the two other paralogs no staining of the myotome was observed all along the antero-posterior axis (Fig. 4C panels c' and j). ptbp3 mRNAs were also broadly detected in the intermediate mesoderm and the lateral plate mesoderm (Fig. 4C panels $i$ and j).

\section{Discussion}

Our data revealed that the three paralogs encoding PTBP1, PTBP2 and PTBP3 have distinct expression profiles. Among the three genes, ptbp1 is the prevalent maternal transcript and it preferentially localizes to the animal hemisphere in UFE and blastula. This temporal and spatial expression pattern suggests that PTBP1 may play important functions in the post-transcriptional regulations that take place during early development. Accordingly, VgRBP60, one of the PTBP1 isoforms, is involved in the transport of the maternal $\mathrm{Vg} 1$ transcript to the vegetal hemisphere of the oocyte (Cote et al., 1999).

At the early gastrula stage, ptbp1 which is considered as a generally expressed RBP is present in only two of the primitive germ layers, the ectoderm and the mesoderm suggesting its early differential expression may play important function in establishing the basic body pattern of the embryo. Since PTBP1 may self regulates through a negative feed back loop that uses alternative splicing to generate a NMD-targeted isoform (Wollerton et al., 2004) it will be of interest to investigate whether the absence of expression in the endoderm results from a transcriptional silencing or is the consequence of a post-transcriptional regulation. Later in development ptbp 1 is widely expressed in many tissues including the dermatome, the intermediate mesoderm, the lateral plate mesoderm and the neural crest that will give rise to numerous cell lineages. Our data also show that neither ptbp1 nor the two other paralogs are expressed in the myotome that will form the skeletal muscles. This observation suggests that the absence of any PTB activity in this tissue is a key determinant to induce skeletal muscle differentiation through the generation of skeletal-muscle-specific splicing patterns and additional post-transcriptional mechanisms. Accordingly, we previously showed that a low level of PTBP1 activity is required in the myotome to produce a muscle specific isoform of the $\alpha$-tropomyosin (Le Sommer et al., 2005). All three family members are expressed in the eye, however, their expression pattern is strikingly non-redundant. While ptbp 1 is restricted to the proliferative $\mathrm{CMZ}$, ptbp2 appears restricted to the neural retina and excluded from the CMZ. Ptbp3 is mainly expressed in the posterior part of the lens. This emphasized the potentially diverse functions of each PTB family members.
Among the three paralogs, ptbp2 displays the more restricted pattern of expression since it is expressed only in the brain, the neural retina, the spinal cord and the intermediate mesoderm. Its expression in different regions of the central nervous system enlightens the key function of this RBP in the establishment and maintenance of the neurogenic program. Its expression in the intermediate mesoderm is shared with the two other paralogs. Since this tissue may give rise to many organs it will be interesting to further investigate whether their expression domains overlap and if so, their functional interplay.

Our analysis also showed that ptbp3 is present in the ventral blood islands which is in agreement with its role in erythroid differentiation (Yamamoto et al., 1999). However, its expression in several non-hematopoietic tissues such as the lens and dermatome also suggests unexplored functions for PTBP3 during development. Strikingly, it is detected in specific subdomains of the brain and the spinal cord as well as in the posterior part of the notochord evoking a possible role in the regionalization of the nervous system. To study further the function of ptbp3 during Xenopus embryogenesis it will be of interest to use functional approaches including morpholino mediated loss of functions experiments.

\section{Materials and Methods}

\section{cDNA clones}

Plasmids containing the full-length cDNA for Xenopus tropicalis ptbp1 (IRBNp992F0722D), ptbp2 (IRBNp992A0837D) ptbp3 (IRAKp961006328Q) (were purchased from imaGenes $\mathrm{GmbH}$. The full length cDNA clone for Xenopus tropicalis sox17b (IRBNp992D099D) was obtained from Source BioSciences LifeScience. Alignment of PTB protein sequences from several organisms was realized using the MAFFT program (Katoh et al., 2002) and the phylogenetic tree was calculated with the Neighbor-Joining method and Poisson estimates and bootstrap sampling using the Archeopteryx software (Han and Zmasek, 2009).The following sequences were used: Homo sapiens ptbp1 NM_002819, ptbp2 NM_021190, ptbp3 NP_001231826; Mus musculus ptbp1 AK053926, ptbp2 AK137348, ptbp3 BC057641; Canis familiaris ptbp1 XM_542215, ptbp2 XM_001381797, ptbp3 XP538790; Gallus gallus ptbp1 AJ851444, ptbp2 XM_422322, ptbp3 XM_424912; Monodelphis domestica ptbp1 XM_542215, ptbp2 XM_001381797, ptbp3 XM_001376071, Xenopus laevis ptbp1a NM_001086700 ptbp1b NM_001090346, ptbp3 BC072378; Drosophila melanogaster PTB AAL14775.

\section{qRT-PCR assays}

To get an independent normalization of qPCR analysis, reporter mRNA luciferase (Promega) was added to frozen embryos (10 pg/ embryo). Total RNA was extracted from $X$. tropicalis embryos at the indicated stages according to Niewkoop and Faber (Nieuwkoop and Faber, 1956) using Tri-reagent (Molecular research center) and genomic DNA contamination was removed by treatment with Turbo ${ }^{\mathrm{TM}}$ DNase (Ambion). RNAs were reverse transcribed using Superscript II reverse transcriptase and random primers following the manufacturer recommendation (Invitrogen). Real-time quantitative RT-PCR analysis was performed on the QPCR 7900 $\mathrm{HT}$ (Applied Biosystems) using the Sybr ${ }^{\mathrm{TM}}$ Green master mix and the following primers: Xenopus odc Forward (fw) tgtgaatgatggcgtgtatg, Reverse (rv) ggtcgaacctttccacaatac; luciferase fw cattcttcgccaaaagcactgtgc, rv agcccataccttgtcgtatccc; ptbp $1 \mathrm{fw}$ ttacaccccaatgcctctt, rv cattgcaagttgagcttggt; $p t b p 2 \mathrm{fw}$ cagcttgcaatgagtcatctta, rv acattgcctcctgtgttgtaa; $p t b p 3$ fw tgaagaagcagctgtcagtatg, rv caatgcagcctgtgctcta. For each primer pair, the PCR product was examined by gel electrophoresis, and its melting curve verified. Ct value obtained for each sample was normalized to the expression level of luciferase reporter (data not shown) or odc mRNA and 2 delta Ct was calculated. Relative level of each mRNA was calculated to 
expression level of ptbp1 mRNA level in UFE normalized to odc mRNA. For each sample, quantifications were done in triplicate.

\section{In situ hybridization and section}

Full length or 3'UTR DIG-labeled antisense and sense riboprobes were generated by in vitro transcription using T7 and SP6 RNA polymerase (Promega), respectively.

Whole-mount in situ hybridization was performed essentially as described in Harland (Harland, 1991). Detection was carried out by anti-DIG Alkaline Phosphatase conjugated antibodies (Roche) and staining was performed with NBT/BCIP (Promega) as the alkaline phosphatase substrate. Embryos were bleached afterwards with $1.2 \%$ hydrogen peroxide in SSC. For more detailed analysis, stained embryos of selected stages were embedded in agar and sectioned at $50 \mu \mathrm{m}$ using a vibratome VT1000S (Leica). Pictures were captured using a Zeiss Axiolab microscope (Leica). To reveal deep expression pattern, fixed UFE and embryos were bisected through the animal-vegetal axis using a razor blade and subjected to in situ hybridization.

\section{Acknowledgments}

We thank Carole Gautier-Courteille and Laurent Richard-Parpaillon for the critical reading of the manuscript and Sylvie Rétaux for her expertise regarding Xenopus neuroanatomy. We thank Claude Sevellec for her help in the realization of the vibratome sections and the platforms of the Biosit research center for the microscopic images and vibratome sections.

\section{References}

ASHIYA, M. and GRABOWSKI, P.J. (1997). A neuron-specific splicing switch mediated by an array of pre-mRNA repressor sites: evidence of a regulatory role for the polypyrimidine tract binding protein and a brain-specific PTB counterpart. RNA 3: 996-1015.

BOURDELAS, A., Li, H.Y., BOUCAUT, J.C. and SHI, D.L. (2004). Identification of distinct genes with restricted expression in the somitic mesoderm in Xenopus embryo. Gene Expr Patterns 4: 695-699.

BOUTZ, P.L., CHAWLA, G., STOILOV, P. and BLACK, D.L. (2007a). MicroRNAs regulate the expression of the alternative splicing factor $\mathrm{nPTB}$ during muscle development. Genes Dev 21: 71-84.

BOUTZ, P.L., STOILOV, P., LI, Q., LIN, C.H., CHAWLA, G., OSTROW, K., SHIUE, L., ARES, M., JR. and BLACK, D.L. (2007b). A post-transcriptional regulatory switch in polypyrimidine tract-binding proteins reprograms alternative splicing in developing neurons. Genes Dev 21: 1636-1652.

COTE, C.A., GAUTREAU, D., DENEGRE, J.M., KRESS, T.L., TERRY, N.A. and MOWRY, K.L. (1999). A Xenopus protein related to hnRNP I has a role in cytoplasmic RNA localization. Mol Cell 4: 431-437.

D'SOUZA, A., LEE, M., TAVERNER, N., MASON, J., CARRUTHERS, S., SMITH, J.C., AMAYA, E., PAPALOPULU, N. and ZORN, A.M. (2003). Molecular components of the endoderm specification pathway in Xenopus tropicalis. Dev Dyn226: 118-127.

HAMON, S., LE SOMMER, C., MEREAU, A., ALLO, M.R. and HARDY, S. (2004). Polypyrimidine tract-binding protein is involved in vivo in repression of a composite internal/3' -terminal exon of the Xenopus alpha-tropomyosin Pre-mRNA. J Biol Chem 279: 22166-22175

HAN, M. V. and ZMASEK C. M. (2009). phyloXML: XML for evolutionary biology and comparative genomics. BMC Bioinformatics 10: 356.

HARLAND, R.M. (1991). In situ hybridization: an improved whole-mount method for Xenopus embryos. Methods Cell Biol 36: 685-695.

KATOH K, MISAWA K, KUMA K, and MIYATA T. (2002). MAFFT: a novel method for rapid multiple sequence alignment based on fast Fourier transform. Nucleic Acids Res 30: 3059-3066

KNOCH, K.P., BERGERT, H., BORGONOVO, B., SAEGER, H.D., ALTKRUGER, A., VERKADE, P. and SOLIMENA, M. (2004). Polypyrimidine tract-binding protein promotes insulin secretory granule biogenesis. Nat Cell Biol 6: 207-214.
LE SOMMER, C., LESIMPLE, M., MEREAU, A., MENORET, S., ALLO, M.R. and HARDY, S. (2005). PTB regulates the processing of a 3'-terminal exon by repressing both splicing and polyadenylation. Mol Cell Biol 25: 9595-9607.

LILLEVALI, K., KULLA, A. and ORD, T. (2001). Comparative expression analysis of the genes encoding polypyrimidine tract binding protein (PTB) and its neural homologue (brPTB) in prenatal and postnatal mouse brain. Mech Dev 101:217-220.

LLORIAN, M., SCHWARTZ, S., CLARK, T.A., HOLLANDER, D., TAN, L.Y., SPELLMAN, R., GORDON, A., SCHWEITZER, A.C., DE LA GRANGE, P., AST, G. and SMITH, C.W. (2010). Position-dependent alternative splicing activity revealed by global profiling of alternative splicing events regulated by PTB. Nat Struct Mol Biol 17: 1114-1123.

MAKEYEV, E.V., ZHANG, J., CARRASCO, M.A. and MANIATIS, T. (2007). The MicroRNA miR-124 promotes neuronal differentiation by triggering brain-specific alternative pre-mRNA splicing. Mol Cell, 27: 435-448.

NIEUWKOOP, P. and FABER, J. (1956). Normal table of Xenopus laevis. Daudin, North-Holland, Amsterdam, The Netherlands.

OHNO S, SHIBAYAMAM, SATO M, TOKUNAGAA, and YOSHIDAN. (2011). Polypyrimidine tract-binding protein regulates the cell cycle through IRES-dependent translation of CDK11 (p58) in mouse embryonic stem cells. Cell Cycle 10:3706-3713.

SAULIERE, J., SUREAU, A., EXPERT-BEZANCON, A. and MARIE, J. (2006). The polypyrimidine tract binding protein (PTB) represses splicing of exon $6 B$ from the beta-tropomyosin pre-mRNA by directly interfering with the binding of the U2AF65 subunit. Mol Cell Biol 26: 8755-8769.

SAWICKA, K., BUSHELL, M., SPRIGGS, K.A. and WILLIS, A.E. (2008). Polypyrimidine-tract-binding protein: a multifunctional RNA-binding protein. Biochem Soc Trans 36: 641-647.

SHIBAYAMA M, OHNO S, OSAKA T, SAKAMOTO R, TOKUNAGA A, NAKATAKE Y, SATO M, and YOSHIDA N. (2009). Polypyrimidine tract-binding protein is essential for early mouse development and embryonic stem cell proliferation. FEBS J. 276: 6658-68.

SPELLMAN, R., LLORIAN, M. and SMITH, C.W. (2007). Crossregulation and functional redundancy between the splicing regulator PTB and its paralogs $\mathrm{NPTB}$ and ROD1. Mol Cell 27: 420-434

SUCKALEJ, WENDLING O, MASJKURJ, JÄGERM, MÜNSTERC, ANASTASSIADIS $\mathrm{K}$, STEWART AF, and SOLIMENA M. (2011). PTBP1 is required for embryonic development before gastrulation. PLOS One 6: e16992.

TAMANOUE Y, YAMAGISHI M, HONGO I, and OKAMOTO H. (2010). Polypyrimidine tract-binding protein is required for the repression of gene expression by all-trans retinoic acid. Dev Growth Differ 52: 469-479.

WAGNER, E.J. and GARCIA-BLANCO, M.A. (2001). Polypyrimidine tract binding protein antagonizes exon definition. Mol Cell Biol 21: 3281-3288.

WANG, C. NORTON, J.T., GHOSH, S, KIM, J., FUSHIMI, K., WU, J.Y, STACK, M.S and HUANG, S. (2008). Polypyrimidine tract-binding protein (PTB) differentially affects malignancy in a cell line-dependent manner. J Biol Chem283:20277-20287.

WESLEY C.S., GUO H, CHAUDHRY K.A., THALI M.J., YIN J.C., CLASON T. and WESLEY U.V. (2011). Loss of PTB or negative regulation of Notch mRNA reveals distinct zones of Notch and actin protein accumulation in Drosophila embryo. PLoS One 6: e21876.

WOLLERTON M.C., GOODING C., WAGNER E.J., GARCIA-BLANCO M.J. and SMITH C.W. (2004). Autoregulation of polypyrimidine tract binding protein by alternative splicing leading to non sense-mediated decay. Mol Cell 13: 91-100.

XU, M. and N. B. HECHT (2007). Polypyrimidine tract binding protein 2 stabilizes phosphoglycerate kinase 2 mRNA in murine male germ cells by binding to its 3'UTR. Biol Reprod 76: 1025-1033

$\mathrm{XU}, \mathrm{M}$. and N. B. HECHT (2008). MSY2 and polypyrimidine tract binding protein 2 stabilize mRNAs in the mammalian testis. Int $J$ Androl 31: 457-461.

YAMAMOTO, H., TSUKAHARA, K., KANAOKA, Y., JINNO, S. and OKAYAMA, H. (1999). Isolation of a mammalian homologue of a fission yeast differentiation regulator. Mol Cell Biol 19: 3829-3841. 


\section{Further Related Reading, published previously in the Int. J. Dev. Biol.}

The zebrafish sf3b1b460 mutant reveals differential requirements for the sf3b1 pre-mRNA processing gene during neural crest development

Min An and Paul D. Henion

Int. J. Dev. Biol. (2012) 56: 223-237

The RNA-binding protein Xp54nrb isolated from a Ca2+-dependent screen is expressed in neural structures during Xenopus laevis development

Isabelle Neant, Nina Deisig, Pierluigi Scerbo, Catherine Leclerc and Marc Moreau

Int. J. Dev. Biol. (2011) 55: 923-931

Regulation and expression of elrD1 and elrD2 transcripts during early Xenopus laevis development

Fida Nassar

Int. J. Dev. Biol. (2011) 55: 127-132

Temporal and spatial expression patterns of Cdc25 phosphatase isoforms during early Xenopus development

Nobushige Nakajo, Yu-Ki Deno, Hiroyuki Ueno, Chihiro Kenmochi, Ken Shimuta and Noriyuki Sagata

Int. J. Dev. Biol. (2011) 55: 627-632

Differential expression of the Brunol/CELF family genes during Xenopus laevis early development

Jingyang Wu, Chaocui Li, Shuhua Zhao and Bingyu Mao

Int. J. Dev. Biol. (2010) 54: 209-214

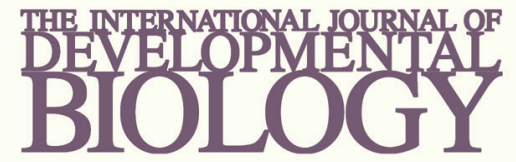

Volume 54 Nos. $6 / 7$
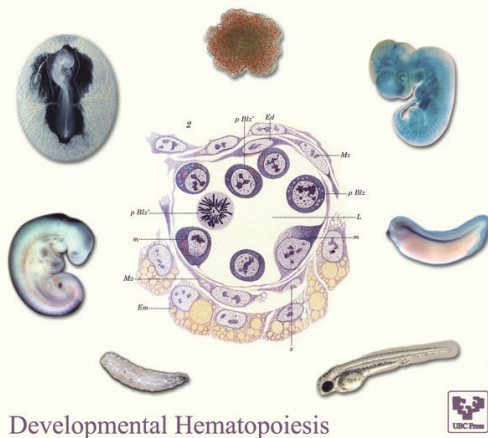

5 yr ISI Impact Factor $(2011)=2.959$
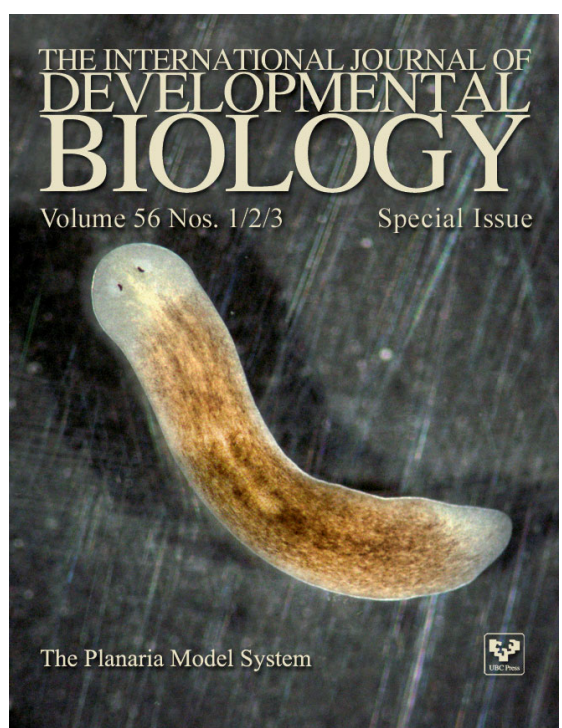
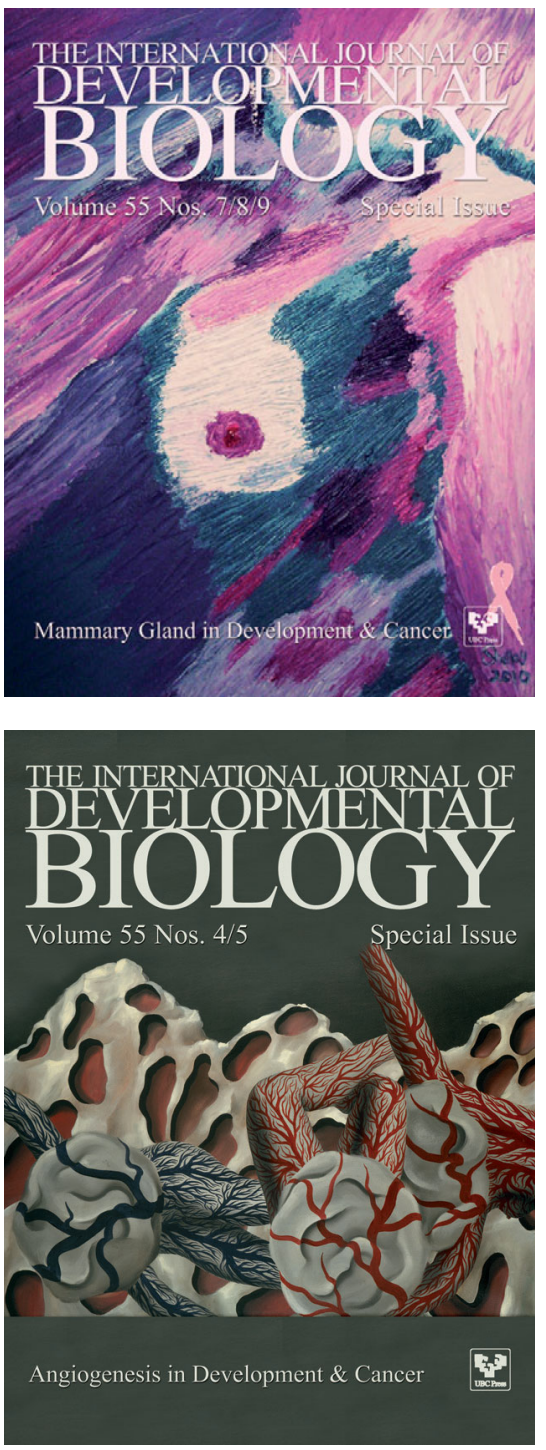